\title{
Public-private partnerships and efficiency in public procurement of primary healthcare infrastructure: a qualitative research in the NHS UK
}

\begin{abstract}
Aim There is growing interest in the contribution of public-private partnerships (PPPs) bridging the shortage of financial resources and management expertise in developing public healthcare infrastructure. However, few studies have evidenced PPPs' ability in increasing efficiency in public procurement of primary healthcare infrastructure. The aim of this study was to assess to what extent PPPs would increase efficiency in public procurement of primary healthcare facilities.

Subject and Methods A qualitative analysis, adopting a realistic research evaluation method, used data collected from a purposive sample of public $(n=23)$ and private sector staff $(n=2)$ directly involved in the UK National Health Service Local Improvement Finance Trust (LIFT).

Results We find a positive association of LIFT helping to bridge public sector capital shortages for developing primary care surgeries. LIFT is negatively associated with inefficient procurement because it borrows finance from private banks, leaving public agencies paying high interest rates. The study shows that some contextual factors and mechanisms in LIFT play a major part in obstructing public staff from increasing procurement efficiency.

Conclusion PPP's ability to increase efficiency may be determined by contextual factors and mechanisms that restrict discretion over critical decisions by frontline public sector staff. Developing their capacity in monitoring PPP activities may make partnerships more efficient.
\end{abstract}

Keywords: Efficiency; Public-Private Partnerships; Primary Care Buildings; LIFT; NHS; UK 


\section{Introduction}

Health systems across the world are increasingly considering engaging the private sector in providing services previously reserved for government. Recently, public and private sector organisations have been encouraged to work in partnership to provide publicly-used health services and physical infrastructure. Public-Private Partnerships (PPPs) are trendy and arguably driven by government's desire to share risks, responsibility, and opportunities in financing aspects of service delivery and activities for designing, constructing and maintaining healthcare facilities. Despite a wealth of evidence about PPPs in health and social care, there is little evidence to show their impact in health outcomes (Hunter et al. 2011). Dickson and Glasby (2010) highlight that: "over time, a series of reviews of partnership literature all conclude that the vast majority of research to date focused on issues of process, not on outcomes" (pp.813-814). This may be due to the complexities of public health embedded with several policies, professionals and organisations (Hunter et al. 2010).

In public administration, the term 'private sector' means the plethora of non-governmental organisations (NGOs) encompassing corporations, voluntary providers, community groups, or individuals whose activities are not directly controlled by government. In Powell and Glendinning's (2002) view, "partnership suggests at least two agencies with common interests working together, in a relationship characterised by some degree of trust, equality and reciprocity" (p.4). The distinction is important because the partners' motives in health differ in ways that influence ability to increase service delivery efficiency.

Broadly, PPPs for health are logical because they concern synergies and complementarities in service delivery. One study in Glasgow shows improvements linked with access to healthcare; and efficiency and effectiveness in service delivery, including health providers', carers' and users' satisfaction with improved health and wellbeing (Fischbacher et al. 2007). This is because whether private partners are for-profit or not-for-profit, together with public organisations, they have unique strengths and weaknesses influencing their ability to deliver specific services. We could argue that effectiveness in service delivery is achieved when both public and private sector partners draw on each other's strengths to complement their individual weaknesses (World Bank 2006). So, the public organisations may be hoping to improve service delivery by bringing in new competencies through partnerships. However, it may require investments in capacity-building for public organisations' staff to be efficient in monitoring the private partners' activities. This might not be a major problem in rich health systems where well developed and experienced private sector partners are more likely to produce benefits than risks in service delivery using complex PPP models.

PPPs for health and wellbeing, therefore, vary depending on the types of risks shared or transferred between the public and private partners. In the UK, although the responsible National Health Service (NHS) retains control over healthcare and strategic services decisions, they have decentralised many aspects of policy-making to health departments in the individual countries (England, Scotland, Northern Ireland and Wales) (Stevens 2004). Subsequently, in England, there is preference of performance improvement policies that de-concentrates decision-making about health provision and management from the Department of Health (DH) to operational levels. We could argue 
that changes within England favour market mechanisms designed to free NHS units, namely Strategic Health Authorities; Foundation Trusts; and Primary Care Trusts (PCTs) from the DH bureaucracy. In particular, PCTs responsible for the lowest level of NHS activities, including related infrastructure development, are encouraged to consider working in partnership with local providers (DH 2001), meaning that some practices could be legitimised under the label of PPPs.

In England, Primary Care Trusts (PCTs) and independent general practitioners (GPs) were responsible for development of primary healthcare surgeries. The PCTs used direct funding from the DH while the GPs used private finance, or grants from the DH passed via the PCTs (Peckham et al. 2005). In 2000, the DH introduced the Local Improvement Finance Trust (LIFT) partnerships, with a mandate to spearhead activities in procurement of primary healthcare surgeries. LIFT operated on the basis of PCTs partnering with local investors to create investment vehicle companies (LIFT companies) that would have a role in mobilising capital needed as well as leading in planning, designing, construction and post-delivery management of new primary healthcare surgeries for lease by the PCTs and occupation by independent GPs (DH 2001). Since then, the DH has not been interested in funding alternative strategies whether directly or through grants in procuring primary healthcare surgeries (Department of Health and Partnerships for Health 2003). Instead, PCTs are expected to adopt LIFT as the default strategy should they wish to improve functionality of primary healthcare in their localities. The term "LIFT" is therefore used in order to recognise the initiative's use of local resources in improving the conditions at local primary healthcare surgeries.

The complexity of the LIFT model of partnership arises from it having features that reflect different public-private partnership (PPP) arrangements. For example, its features mimic the buildoperate-transfer, design-build-finance-operate, lease-purchase PPP models occasionally used within the NHS. This creates problems in seeking to understand LIFT's ability in being an efficient and effective strategy for transferring or sharing procurement risks between the partners. While the different PPP models reflected in it may have more or less similar arrangements for managing risks, the LIFT model differs in how it handles the ownership of PPP buildings. LIFT changes how primary healthcare buildings are procured but does not substitute for the government role concerning decision-making and governance in procurement activities. The DH and other strategic government departments still have significant say and role in procurement of primary healthcare surgeries, probably in order to optimise efficiency in use of public funds.

Perhaps the underpinning assumption in adopting LIFT is the DH perception that PPP-led procurement may facilitate local PCTs in being more efficient procurers (DH 2001). There is an implied association of private sector participants in LIFT with having skills and expertise to mobilise finance, and the ability to manage initiated projects. Already, under LIFT, the National Audit Office (2005) and King's Fund (2008) see these factors facilitating timely delivery of desired surgeries within budget and without compromised quality in the NHS. It chimes with other analysts of healthcare PPPs who see improvements in procurement as the result of the preferred private sector partners being proven experts in their allocated roles (Perrot 2006; Pollit and Bouckaert 2000).

While Hunter et al. (2011) remind us that "experiments with different forms of partnership (...) appear necessary to tackle complex public health challenges" (p.125), few studies have evidenced 
PPPs' ability to increase efficiency when used in developing public infrastructure. The aim of this study was to assess to what extent LIFT would increase efficiency in the development of primary healthcare surgeries.

Our choice of studying the public-private partnership (PPP) model is grounded on criticism that it has complex governance arrangements which potentially restrict achieving the efficiency that is expected to benefit the involved PCTs (Beck et al. 2009). The study may help in dispelling the myth about PPPs of this nature being effective at solving problems in government-led development of primary healthcare surgeries. We anticipate generate knowledge about PPP contextual factors and mechanisms that may restrict achievement of efficiency, hoping that they may provide caution to promoters against taking for granted that efficiency will be achieved simply because the preferred private partners are experts in their allocated roles. It helps in identifying areas for revision on PPP guidance if they facilitate the effectiveness of public agencies in being more efficient in their procurement activities.

\section{Methods}

Study design

A qualitative research using a realist research evaluation approach was used in this study. Qualitative research may relate to the subjective world but provides helpful insights into social, emotional and experimental phenomena (Giacomini and Cook 2000). Its essence lies in drawing out “(...) understandings and perceptions, to explore the features of settings and culture (...)" in order to understand linkages between phenomena, processes and outcomes (CRD 2008, p.221). Ailinger (2003) argues that qualitative evidence "seeks to analyse the complexity of human phenomena in naturalistic settings and form a holistic perspective" (p.15).

Especially when inspired by realism in the analysis, it may be an effective way of considering the views of major stakeholders, including people directly involved or affected by phenomena whether as practitioners, providers or service-users - in order to develop a holistic understanding of how PPPs work based on their experiences, preferences and priorities (Evans 2002). According to Pawson and Tilley (2004), realist evaluation is a useful approach to explaining whether resources reserved for public programmes are actually used to maximise public value. The approach prioritises exploring key aspects such as what works, why and how, as well as identifying the groups that benefit, and circumstances under which efficiency is affected.

\section{Study participants}

The following approaches were utilised: (a) Primary Care Trusts (PCTs) - Two geographically neighbouring PCTs were case-studied before they were phased out in 2012. They were chosen based on their homogeneous population; health and social profiles; and having similar problems in conditions of their primary healthcare facilities. Also very important was their experience as pioneers of LIFT 
partnerships in London; (b) LIFT company: Only one LIFT company is shared between the Primary Care Trusts (PCTs). Focusing on one rather than a series of LIFT companies would facilitate deeper analysis in a limited time; (c) LIFT premises: The portfolio of buildings developed through LIFT within the PCTs allowed enquiries at five premises owned and managed by the same LIFT company. The premises included the NHS' first ever LIFT building. The building would help us in reaching informants well experienced in LIFT, whether at PCT or premises levels; (d) Informants: 25 informants drawn from the PCTs, LIFT company, and LIFT premises contributed data central to this analysis. Their demographic attributes are given in Table (1), which also shows that the majority of informants $(n=23)$ were PCT employees who could be construed to represent public sector views. The two informants from the LIFT company represent private sector views. In light of our thrust to explain the efficiency contribution of PPPs in developing public primary healthcare infrastructure, this distribution of informants may be viable for protecting us from relying on private sector perspectives that are less driven by public sector objectives.

[Table 1 about here]

Such distribution of participants facilitated getting comparative perspectives about the LIFT partnership model from those who experienced it within or outside of the NHS. Significantly, the participants' 150 combined years of experience in LIFT was helpful in validating the findings.

Data collection

We employed the following methods to gather the information: (a) In-depth interviews: Semistructured interviews were conducted using topic guides comprising 40 investigative questions and ideas around participants' beliefs and attitudes, and their interpretation of social, cultural, behavioural and emotional aspects in LIFT that influence how they achieve its objectives and perform their roles within the PCT areas; (b) Observation: Tours of the selected buildings were conducted in order to observe and gather data for confirming the feedback in interviews with premises administrators and lead GPs. The data would help us in judging whether, and explaining how, LIFT is a step improvement in solving problems in public procurement of primary care buildings; (c) Documentary reviews: Complementary data relating to guidance and procedures, expected outcomes, and best practices in LIFT were extracted from documents identified from DH-linked websites or provided by informants, unless deemed too sensitive for sharing.

Data analysis

Both researchers were involved in analysis of the field data. We prioritised a systematic approach in enquiring to capture the real-world experiences of connections between LIFT contexts, mechanisms, and outcomes that characterise health PPPs. Capturing the real-world experiences was deemed to be helpful as it would integrate different analytical strategies in explaining efficiency by systematically 
identifying and summarising the main recurrent and/or most important themes contained in LIFT documents, in-depth interviews, and observations at LIFT buildings (Mays et al. 2005). In order to capture the relevant data, we first sought agreement on how to identify LIFT contexts and mechanisms, because they are central to our interpretations of activities in its implementation.

We adopted the generic explanation that context would describe the social, economic and cultural conditions within which LIFT is implemented. Such conditions are external to a LIFT and either facilitate or constrain the partnership in producing the expected benefits in procurement of desired buildings (Marchal et al. 2010; Pawson and Tilley 2004). In the same vein, mechanisms would be identified as opportunities plus ideas that are introduced through the LIFT PPP model. They are like in a "black box" because they are not physically seen, yet their being internal to LIFT may influence it to produce reported outcomes (Marchal et al. 2010). This approach to data analysis helped us in explaining LIFT aspects that worked to benefit whom, in what circumstances, and how, among its participating groups. It facilitated a systematic evaluation of the partnership model's contexts and mechanisms to understand how they supported staff at the PCTs in achieving efficiency expected in procurement of primary healthcare surgeries.

Evidence extraction and coding

The evidence about how LIFT contexts and mechanisms contributed to efficiency in procurement was verified through analyses of data in official documents, interview transcripts, and notes on observations at the buildings. All these data sources were uploaded for management and analysis using NVivo 9.0. The software offered the advantage of having the capacity to store and efficiency to analyse multiple documents containing large amounts of textual data. The documents were likely to present challenges in analysis if we had chosen to handle them manually. Using NVivo facilitated a systematic approach to coding complex and broad issues about efficiency contained in documents from different sources.

Within NVivo, the authors $(n=2)$ thematically analysed factors that impact participant efficiency in achieving LIFT objectives within the Primary Care Trust (PCT) areas. This involved retrieving data to identify, interpret and manipulate ideas and opportunities that explain either efficiency or inefficiency. As Ritchie et al. (2004) suggest, thematic analysis helps to synthesise the evidence located, which "involves discovering, interpreting and reporting patterns and clusters of meaning within the data" (p.271). The data and ideas identified evidence for how the LIFT model of PPPs responded to the influence of contextual factors and mechanisms through which its activities are implemented. We then assigned all such descriptors of how LIFT responded to influence of the factors surrounding its implementation to codes for evidence about efficiency.

The coding used possibly does not provide exhaustive explanations for efficiency, because the obscurity of some of LIFT's critical issues in official documents risked occasional differences in reasoning them out or interpretation for coding. This was addressed through consensus by the two authors to merge, discard, or create new codes in order to retain those that closely matched our agreed interpretation and indicators of efficiency described in official documents and those mentioned in the interviews. We were particularly interested in identifying how to understand individuals or professional 
groups' experiences and perceptions about PPP mechanisms and contextual factors that most facilitate their effort to efficiently develop and manage improved primary healthcare buildings.

\section{Measuring efficiency}

Notwithstanding the reported minor differences in coding, the two analysts had a shared interpretation of efficiency as described by a PPP's ability to deliver against its intentions at minimum cost or inconvenience of intended beneficiaries. Thus the analysts agreed to understand efficiency in LIFT as best explained by how the partners (PCTs, DH, and LIFT company) and people affected (PCT and LIFT company staff) interpreted the concept, because their interpretations incentivise them to behave in ways that may influence the ability to meet objectives of the PPP. Whether the ideas originated from LIFT documents, interview transcripts or observation notes, we reflected our agreement on interpreting efficiency in data extraction and coding within NVivo.

Much of the interpretation of efficiency in this case therefore prioritises the generic rather than the economic explanations. It does not mean that technical and allocative efficiencies are unimportant measures in interpreting PPP activities. We only preferred the generic and non-quantitative interpretation in the analysis because of the interest in introducing realism that is needed for a better judgement about success in PPPs by explaining causation between activities, outcomes and benefits.

\section{Ethical considerations}

The study was carried out in 2008 upon gaining permission from the UEL Ethics Committee on the grounds of being supported by the concerned PCT management boards and LIFT company.

Participants' anonymity and confidentiality were maintained throughout the study.

\section{Results}

The findings from thematic analyses of activities in implementing the LIFT model of PPPs identified five major categories of efficiency areas:

- Dimensions of quality of buildings

- Increasing public value in procurement

- Risk management

- Competition and diversity

- $\quad$ Service integration

The study reveals that delivering physical infrastructure, LIFT also seeks to improve the quality of primary healthcare surgeries by increasing the participation of GPs in their development; and diversity in the developers as well as other experts in managing associated risks. The ways through which these objectives are achieved significantly influence the PPP model's ability to increase efficiency in procurement activities. The findings further reveal useful efficiency indicators as concerning 
configurations of contextual factors and mechanisms that support public agencies in achieving broader healthcare objectives, commensurate with public money invested in public-private partnerships (Table 2).

[Table 2 about here]

Dimensions of quality of buildings

While we concur with other analysts (King's Fund 2008) that partnerships make progress in meeting patient preference for improved facilities without straining public budgets, we note that people directly affected by partnerships prefer measuring quality at the buildings along indicators other than mere physical outlook and cleanliness. They are interested in relevance of the buildings' plans to local healthcare needs and making sure that the long-term value of buildings is commensurate with mortgages paid, because pressure to meet construction deadlines may mean that locales are given short lead time for providing feedback. It is considered a sign of inefficiency that reduces the quality of buildings when measured according to the relevance of their plans to local needs.

(...) short deadlines to provide meaningful feedback on the plans resulting in tenants being sometimes consulted after the work is done and there is no scope to reverse anything.

- Risk, Health \& Safety Director

The findings show that efficiency is also influenced by effectiveness of procedures followed. For example, sufficiently engaging especially Primary Care Trust (PCT) staff who are experienced in procurement is perceived to increase efficiency when they give input in designing their buildings. LIFT participants associate it with inefficiency because of perceived insufficient engagement with a broader spectrum of experienced PCT staff in designing the buildings. General Practitioners (GPs) who are delegated this role are less likely to prioritise the PCT's aspirations in reducing procurement costs. What is more, the DH officials robustly enforce compliance with PPP procedures, risking reduced efficiency when PCT staff are restricted in initiating ideas for getting maximum benefits from LIFT.

Increasing public value in procurement

It is considered good practice that LIFT partnerships prioritise efficiency in activities for delivering and maintenance of buildings. The efficiency achieved helps optimise value for public money invested into its activities. However, some PCT staff challenge this finding by arguing that fixation with efficiency drives the LIFT company and its contractors to reduce the quality of inputs in construction and maintenance of buildings, risking reduced lives or residual values of LIFT buildings. An administrator reflected that PPPs may increase public value in their early days but over time, this is questionable.

(...) we are not sure whether our LIFT company is now complacent. It is clear that our expectations on maintenance of buildings are not being met because even small jobs like replacing lights and door locks are never done promptly yet we pay rent above market rates. 
It is also seen as an indicator of inefficiency that Primary Care Trusts (PCTs) and their staff lose valuable time and resources on partnership procedures perceived to be of little relevance to solving problems in discharging their responsibilities. A partnership's ability to increase efficiency could be significantly reduced if time and material resources lost to convoluted procedures are factored in for consideration side-by-side with sustainability. This is important, given the duration of most publicprivate partnership (PPP) contracts for healthcare infrastructure development.

\section{Risk management}

One significant finding suggests that a better way of rating the ability of PPPs to increase efficiency in procurement activities should account for adverse effects of some of their mechanisms. In this study, managers at the PCTs advise that efficient risk management is achieved only if PPP companies are able to mobilise cheaper and affordable finance for delivering buildings in the absence of government funding. They seriously criticise LIFT companies for not being helpful in increasing efficiency in procurement, because their propensity to borrow expensive finance from private banks drives them to pass over high interest rates to the PCTs in the form of inflated rent for buildings. Overall cost may increase due to government interference in some procedures. For example, one lead GP considered it inefficient, saying that:

(...) the LIFT company spent $£ 500,000$ on tender preparations alone because they were forced by DH officials to involve too many consultants. - One lead GP

Another issue about effective risk management is the concern that efficiency is missed because private partners do not always respect their share of agreed-upon risks. PCTs adopting LIFT partnerships report being hard-pressed to do self-help activities in order to have continuity of service when LIFT companies are not forthcoming in delivering some of their expected roles. Furthermore, in PPPs like the LIFT model, public agencies tend to always retain responsibility for unpredictable or difficult-to-handle risks like demand for buildings. Thus, they may miss important benefits to accrue if PPP companies and their contractors were to handle the risks as the experts.

Competition and diversity

In theory, PPPs are sold as a strategy for promoting competition in development and post-delivery management of healthcare infrastructure. However, our analysis of LIFT reveals that they are neither efficient nor effective at increasing public sector capacity in developing improved facilities. Although LIFT employs diverse service contractors for different roles, competitive hiring is mainly on financial cost considerations, at the expense of skills and experience in health.

What is more, LIFT operates on the basis of monopoly role in some geographic areas, ostensibly to attract resourceful private partners. And this is another putative factor for reduced 
efficiency in its implementation. Barriers to competition make the companies complacent in their activities while preventing public partners from gaining the benefits associated with competition. On one hand, the small size and capacity of the LIFT company are believed to prevent it from satisfying demand for improved GP surgeries when working alone. On the other hand, having a small PPP company is perceived to be:

(...) more efficient than bureaucratic structures of the government and the Board's expertise and skills protect the PCT from problems of monitoring a bigger company. - Estate \& Facilities Director

Service integration

We found that public-private partnerships (PPPs) may partly help in integrating primary care services for patient convenience. Unlike traditional procurement methods, LIFT prioritises buildings that are suitable for offering a range of care and health promotion services. GPs using LIFT buildings consider them efficient in reducing administrative overheads. Focus is given to central premises occupied by multiple providers, rather than paying for the same activities in different places. Another indicator for efficiency concerns having dedicated administrators at the premises. This helps by relieving the GPs of administrative duties, so that they focus on patient care.

Nonetheless, efficiency may be reduced if independent GPs based at the same PPP buildings do not value linking together or joining with public sector-initiated programmes. We found it is hard for PPPs to increase efficiency by encouraging more care providers to relocate to central premises. High rent and lack of clarity about financial gains may obstruct DH agencies from convincing GPs owning their own buildings to prefer PPP buildings. It is possible that some of the inefficiencies are explained by participants' initial inexperience in using PPP.

Restrictions on ability to increase efficiency

A holistic judgement about efficiency required us to identify and examine key factors that hinder increased efficiency in PPP activities. Themes about supporting DH units in infrastructure planning; community involvement; prioritising efficiency in activities; risks transfer and sharing between partners; and risk assumption service contractors emerged as the key limiting factors (Table 3).

[Table 3 about here]

The selling point of partnerships is said to be their helpfulness in improving public infrastructure that is critical in increasing patient experience (King's Fund 2008; Department of Health and Partnerships for Health 2003). The pledges, however, are only achieved if DH officials offer sufficient technical support for their agencies to be effective in planning strategic infrastructure within their areas. It involves them facilitating exchange of ideas on partnerships so that the private partners 
are able to share essential information for translating their knowledge into projects that solve problems in procurement.

These efficiency-boosting factors are perceived to be missing in LIFT, where progress is further restricted by communities and PCT staff having no voice in decisions about how to develop their own healthcare facilities. PCTs' managers are particularly concerned that they had

“(...) no choice but to accede to LIFT because the DH would not fund any other procurement route. We are not aware of the options considered by the DH nor the criteria used to judge LIFT's suitability for our needs." - Finance Director

The government had hoped that the PCTs would increase efficiency if new GP surgeries were the result of agreement between the LIFT company, PCT managers, and local communities. Yet due to missing meaningful inputs or their not being respected, some buildings fail to reflect local priorities or their unique circumstances. Unsurprisingly, therefore, LIFT is not judged as the efficient way of developing primary healthcare infrastructure. What is also apparent is the role played by private partners in handling risks in development and post-delivery management of LIFT buildings. Efficiency is reduced where the involved private partners and service contractors fail to respect their share of important risks. Especially when too fixated on efficiency, they may reduce costs by hiring cheap labour, using cheap inputs in construction, delaying repair jobs, or being inflexible to adapting buildings according to the needs of tenants. So to a larger or lesser extent, partnerships' ability to increase efficiency may be limited by participants' business approaches and how they interpret their role in the partnership. These factors exacerbate inefficiencies and increase costs if they reduce individual commitment to the partnerships' intentions.

\section{Discussion}

This study of LIFT partnerships identified the critical dimensions of efficiency in developing healthcare infrastructure as: (i) ability to increase the quality of buildings; (ii) drive to maximise value for public money; (iii) commitment to risk management; and, (iv) valuing integration of services delivered through competition. As Hunter et al. (2011) suggest, these factors are embodiments of attitudes, sectoral cultures and behaviours that support partnerships in improving or promoting participation in planning and delivering strategic public health services. Increased efficiency may be achieved with effective transfer of procurement risks from the public to management by the private partners, especially where they have a leadership role including managing service contractors (World Bank 2006; Fitzsimmons et al. 2009).

There is need to appreciate the existence of confounding factors other than public-private partnership (PPP) features that influence efficiency (Peckham et al. 2005). As with LIFT, partnership guidance that merely encourages or duplicates existing public agency practices is likely to be less effective in increasing efficiency than guidance introducing new ideas and opportunities. Perhaps PPP beneficiaries see no benefits in sticking with partnerships whose guidance or activities are similar to previous routines for developing or managing healthcare infrastructure. The role of behaviour, 
attitudes, and public and private provider differences in approaches to service delivery becomes clear in this case. The factors may explain participant acquaintance with challenges in translating PPP guidance into reality. In LIFT, familiarity with the guidance is associated with reasonable progress in making activities more efficient, because it may facilitate partnership mechanisms in retaining their usefulness in producing desired outcomes (Beck et al. 2009).

Conversely, insufficient acquaintance with complex guidance may influence public-private partnership (PPP) leadership to assess efficiency by only including easy-to-measure expenses potentially only those incurred by private partners - ignoring non-quantitative ones by public agencies. It risks understating costs and portraying PPPs as efficient, even when neither increasing public value nor proving to be a viable procurement method in future.

According to Saltman et al. (2007), PPPs are most efficient where local staff enjoy more discretion. Similarly, Hunter et al. (2011) argue that to become effective, "it is also desirable to be specific about the type of partnership being discussed, the context in which it is to operate, and what the success criteria are supposed to be, assuming they have been set up and agreed in the first place" (p.27). In this study, inefficiencies are partly explained by insufficient freedom by local staff in influencing some of LIFT's critical activities, like hiring service contractors and decisions about using funds set aside to assist GPs in relocating to new premises (King's Fund 2008). Crucially, it requires effective transfer of critical risks for management by private partners to achieve efficiency (World Bank 2006). Yet in most PPP types including LIFT, efficiency benefits are missed because the private partners have weak technical capacity for handling important risks (Pollock 2006). Perrot (2006) explains it as the result of assessing private partners' technical capacities only at the stage of their appointment. It risks not accounting for changes in circumstances that may impact the ability to manage unforeseen yet major risks. Pollit and Bouckaert (2000) argue that PPPs achieve more with minimum resources by using diverse mechanisms in reducing costs and management of risks in procurement. This study also adds knowledge regarding challenges which the individual participants in partnerships face in translating these efficiency pledges into reality.

Much of the discourse on partnerships emphasises economic arguments, to highlight how private partners improve procurement activities by handling economic factors that impact technical and allocative efficiencies better than their public sector counterparts. This study introduces new evidence based on realism about the role of social, cultural and behavioural factors. The factors give PPP participants, whether public or private partners, or service contractors, the incentive to behave and act in ways that may impact on meeting PPP objectives. This paper therefore suggests that in assessing PPP efficiency, better judgement is feasible if questions are asked about which participants benefit from what PPP activities, and how they benefit.

It may be considered a limitation that our ideas derive from a single case-study involving one partnership company. But the institutions and individuals have rich experience, which is helpful in validating our ideas and explaining ways in which partnerships may facilitate public agencies towards efficiency. Hence the study adding the evidence that PPPs' ability to increase efficient healthcare infrastructure development is perhaps overstated, because it is quantitatively assessed in ways that focus on economic aspects without accounting for attitudinal, cultural and behavioural factors. 
There are two important implications for policy and practices that we discuss in relation to efficiency in service delivery: first, differences in approaches to achieving efficiency in service delivery between public and private sectors. In LIFT, we learn that PPP companies are not likely to adapt their business principles as expected by public agencies. They do not prioritise community engagement, increased competition or taking over extended risks. Prolonging project times tends to increase costs. Yet PPPs should ensure that public agencies do not miss efficiency benefits by adjusting their activities to suit circumstances in health (Perrot 2006). Second, adopting a holistic explanation of efficiency in PPP activities. Good practice uses a broader scope in assessing efficiency. Rather than using monetary terms alone, some of the important influential factors, indirect or intangible, should be considered.

Hunter et al. (2001) argue that system thinking may "demonstrate[s] that managing complex adaptive systems demands a new mind-set that may be more focused on improving what can be done rather than trying to meet a specified target or goal that may be unrealistic, unattainable and just wrong” (p.28). Currently, practice in LIFT is problematic because it considers only easy-to-measure direct and current costs. A realistic assessment of ability to increase efficiency should also consider future interest rates and payments for utilities. Being transparent about other PPP activities that may influence the gains by public agencies over time is also important.

\section{Conclusion}

Public-private partnerships (PPPs) are acknowledged to be a step improvement in infrastructure development. In particular, LIFT is perceived to be helpful in bridging shortages of public capital resources, skills and expertise in improving primary healthcare surgeries. Nevertheless, opinion is divided between the proponents and those advocating conventional procurement methods about the ability of partnerships to increase efficiency in activities for translating their pledges into reality. Our analysis shows that the role of contextual factors and mechanisms in influencing efficiency, as well as the conditions under which LIFT is implemented and ways through which some of its activities are done, may work individually or together to influence efficiency in activities that maximise benefits. We conclude that to bring positive contributions to increase efficiency in public procurement depends upon the degree of bureaucracy involved in terms of decentralisation - decision-making, governance, accountability - and strong, effective and formal partnerships.

\section{Acknowledgements}

This article draws on a public health research project hosted by the IHHD at UEL. We thank the study participants and the health officials for their support and cooperation during the study. We would also like to thank Dr Patrick Tobi, University of East London UK, and Dr Colin Thunhurst, Coventry University UK, for their insightful comments in preparing this paper. 